\title{
BOUNDEDLY COMPLETE $M$-BASES AND COMPLEMENTED SUBSP.ACES IN BANACH SPACES
}

BY

\author{
WILLIAM J. DAVIS AND IVAN SINGER
}

\begin{abstract}
Subsequences of boundedly complete $M$-bases need not be boundedly complete. An example of a somewhat reflexive space is given whose dual and one of whose factors fail to be somewhat reflexive. A geometric description of boundedly complete $M$-bases is given which is equivalent to the definitions of V. D. Milman and W. B. Johnson. Finally, certain $M$-bases for separable spaces give rise to proper complemented subspaces.
\end{abstract}

A sequence $\left(x_{n}\right)$ in a Banach space is called an M-basis (or Markushevich basis) of $E$ if $\left(x_{n}\right)$ is complete in $E$ (i.e., the closed linear span $\left[x_{n}\right]$ of $\left(x_{n}\right)$ is the whole space $E$ ) and if there exists a total sequence of functionals $\left(f_{n}\right) \subset E^{*}$ (i.e., $\left.\left\{x \in E \mid f_{n}(x)=0(n=1,2, \ldots)\right\}=\{0\}\right)$ such that $\left(x_{n}, f_{n}\right)$ is a biorthogonal system (i.e., $f_{i}\left(x_{j}\right)=\delta_{i j}$ for $i, j=1,2, \ldots$ ); obviously, $\left(f_{n}\right)$ is uniquely determined. The sequence $\left(x_{n}\right)$ is called a basis of $E$ if for every $x \in E$ there exists a unique sequence of scalars $\left(a_{n}\right)$ such that

$$
x=\sum_{i=1}^{\infty} \alpha_{i} x_{i} .
$$

It is known [2] that every basis is an M-basis, with $f_{n}(x)=\alpha_{n}\left(x=\sum_{i=1}^{\infty} \alpha_{i} x_{i} \in E\right.$, $n=1,2, \ldots)$, which are called the coefficient functionals.

A basis $\left(x_{n}\right)$ of a Banach space $E$ is called boundedly complete if the relation

$$
\sup _{n}\left\|\sum_{i=1}^{n} \alpha_{i} x_{i}\right\|<\infty
$$

implies that $\sum_{i=1}^{\infty} \alpha_{i} x_{i}$ converges in $E$. It is known $([18],[3])$ that $\left(x_{n}\right)$ is a boundedly complete basis of $E$, with the coefficient functionals $\left(f_{n}\right)$, iff the canonical mapping $\phi$ of $E$ into $\left[f_{n}\right] *$ (i.e., the mapping defined by $[\phi(x)](f)=$ $f(x)$ for all $\left.x \in E, f \in\left[f_{n}\right]^{*}\right)$ is an isomorphism of $E$ onto $\left[f_{n}\right]^{*}$. The notion of boundedly completeness has been extended to $M$-bases in several different ways; most of them equivalent to this property of the canonical mapping $\phi: E \rightarrow\left[f_{n}\right] *$.

Received by the editors June 17, 1971 and, in revised form, December 10, 1971 .

AMS (MOS) subject classifications (1970). Primary 46B10, 46B15.

Key words and phrases. Basis, Markushevich basis, reflexivity, projection, complemented subspace, somewhat reflexive. 
We shall say that an M-basis, $\left(x_{n} ; f_{n}\right)$, of $E$ is boundedly complete if $\phi$ is an isomorpbism onto $\left[f_{n}\right]^{*}$. In $\$ 1$ of the present note we shall disprove a claim of V. D. Milman [21] on boundedly complete M-bases, which, if it had been true, would have provided an affirmative answer to a problem of [4] on boundedly completeness. In $\$ 2$ we consider the existence of reflexive subspaces in Banach spaces. We also answer some questions concerning the somewbat reflexive spaces of Herman and Whitley [10]. In $\$ 3$ we introduce a new geometric characterization of boundedly complete $M$-bases. Finally, in $\$ 4$ we examine complemented subspaces in separable Banach spaces arising from certain $M$-bases.

1. The existence of boundedly complete basic sequences. In [4] the question was raised whether every separable conjugate Banacb space $E^{*}$ contains a subspace with a boundedly complete basis (with no assumption of separability on $E^{*}$ the question was raised in [25, Problem 3.8] and [22, Problem 3]); by subspace we shall mean, throughout this note, closed linear subspace. Since the first version of this paper, Johnson and Rosenthal [16] have given an affirmative answer to the problem. Earlier, V. D. Milman [21] claimed that the answer to this question is affirmative, via the following argument: Since $E^{*}$ is separable, there exists, by a result of Gapoškin and Kadec [7], a biorthogonal system $\left(x_{n} ; f_{n}\right)$ such that $\left[x_{n}\right]=E$ and $\left[f_{n}\right]=E^{*}$. Then $\left(f_{n}\right)$ is a boundedly complete M-basis of $E^{*}$. Since $\left[x_{n}\right]=E,\left(f_{n}\right)$ contains $([17],[21])$ a subsequence $\left(f_{n_{j}}\right)$ which is a basic sequence (i.e., a basis of its closed linear span $\left.\left[f_{n_{j}}\right]\right)$. Now, Milman claims [21, Proposition 3.4b] that every subsequence of a boundedly complete $M$-bas is is a boundedly complete $M$-basis of its closed linear span and hence $\left[f_{n_{j}}\right]$ is a subspace of $E^{*}$ with the desired property, i.e., having a boundedly complete bas is $\left(f_{n_{j}}\right)$. Firstly, we want to remark that this claim is false, even when $\left(f_{n_{j}}\right)$ is a basic sequence, as shown by

Example 1. Let $E=c_{0}$ and let

$$
\begin{aligned}
x_{2 n-1} & =e_{2 n-1}-2^{n} e_{2 n}+2^{n+1} e_{2 n+2} & & (n=1,2, \ldots), \\
x_{2 n} & =2^{n} e_{2 n} & & (n=1,2, \ldots), \\
f_{2 n-1} & =b_{2 n-1} & & (n=1,2, \ldots), \\
f_{2} & =b_{1}+1 / 2 b_{2}, \quad f_{2 n}=-b_{2 n-3}+b_{2 n-1}+\left(1 / 2^{n}\right) b_{2 n} & & (n=2,3, \ldots),
\end{aligned}
$$

where $\left(e_{n}\right)$ is the unit vector basis of $c_{0}$ (i.e., $\left.e_{n}=\{\underbrace{0, \ldots, 0}_{n-1}, 1,0, \ldots\}\right)$ and $\left(b_{n}\right)$ the sequence of coordinate functionals on $E=c_{0}$ (i.e., $b_{n}(x)=\xi_{n}$ for all $\left.x=\left(\xi_{j}\right) \in E\right)$, hence $b_{i}\left(e_{j}\right)=\delta_{i j}(i, j=1,2, \ldots)$. Then $\left(x_{n} ; f_{n}\right)$ is a biorthogonal system. Furthermore, since $e_{2 n}=\left(1 / 2^{n}\right) x_{2 n}, e_{2 n-1}=x_{2 n-1}+x_{2 n}-x_{2 n+2}$ $(n=1,2, \ldots)$ and, since $\left[e_{n}\right]=E$, we have $\left[x_{n}\right]=E$. Similarly, since $b_{2 n-1}=$ 
$f_{2 n-1}, b_{2}=2\left(f_{2}-f_{1}\right), b_{2 n}=2^{n}\left(f_{2 n}-f_{2 n-1}+f_{2 n-3}\right)(n=2,3, \ldots)$ and, since $\left[b_{n}\right]=E^{*}$, we have $\left[f_{n}\right]=E^{*}$, making $\left(f_{n}\right)$ boundedly complete. However, the subsequence $\left(f_{2 n}\right)$ of $\left(f_{n}\right)$ is a basic sequence which is not boundedly complete. Indeed, for any scalars $a_{1}, \cdots, a_{n}$ we have

$$
\begin{aligned}
\left\|\sum_{i=1}^{n} \alpha_{i} f_{2 i}\right\| & =\left\|\alpha_{1}\left(b_{1}+1 / 2 b_{2}\right)+\sum_{i=2}^{n} \alpha_{i}\left(-b_{2 i-3}+b_{2 i-1}+\frac{1}{2^{i}} b_{2 i}\right)\right\| \\
& =\left\|\sum_{i=1}^{n-1}\left(a_{i}-\alpha_{i+1}\right) b_{2 i-1}+\alpha_{n} b_{2 n-1}+\sum_{i=1}^{n} \frac{1}{2^{i}} a_{i} b_{2 i}\right\| \\
& =\sum_{i=1}^{n-1}\left|\alpha_{i}-a_{i+1}\right|+\left|\alpha_{n}\right|+\sum_{i=1}^{n} \frac{1}{2^{i}}\left|\alpha_{i}\right|,
\end{aligned}
$$

whence, for any scalars $\alpha_{1}, \cdots, \alpha_{n+1}$,

$$
\left\|\sum_{i=1}^{n} \alpha_{i} f_{2 i}\right\| \leq \sum_{i=1}^{n}\left|\alpha_{i}-\alpha_{i+1}\right|+\left|\alpha_{n+1}\right|+\sum_{i=1}^{n+1} \frac{1}{2^{i}}\left|\alpha_{i}\right|=\left\|\sum_{i=1}^{n+1} \alpha_{i} f_{2 i}\right\|,
$$

and therefore, $\left(f_{2 n}\right)$ is a "monotone" basic sequence [3]. Finally, for $\alpha_{1}=\cdots=$ $a_{n}=1$, we have $\left\|\sum_{i=1}^{n} f_{2 i}\right\|=1+\sum_{i=1}^{n}\left(1 / 2^{i}\right)(n=1,2, \ldots)$, whence $\sup _{n}\left\|\sum_{i=1}^{n} f_{2 i}\right\|$ $=2$, but $\sum_{i=1}^{\infty} f_{2 i}$ is not convergent, since $\left\|f_{2 n}\right\|>1 \quad(n=1,2, \ldots)$, and thus $\left(f_{2 n}\right)$ is not boundedly complete.

It is interesting to see what conditions on a biorthogonal system $\left(x_{n} ; f_{n}\right)$ such that $\left[x_{n}\right]=E,\left[f_{n}\right]=E^{*}$ will guarante that every basic subsequence $\left(f_{n_{j}}\right)$ of $\left(f_{n}\right)$ is boundedly complete. Standard arguments show that, if $\left[f_{n_{j}}\right]$ is $\sigma\left(E^{*}, E\right)$-closed, then $\left(f_{n_{j}}\right)$ is boundedly complete (if $\left[f_{n}\right]=E^{*}$ ). Hence, since clearly $\left[f_{n_{j}}\right] \subset$

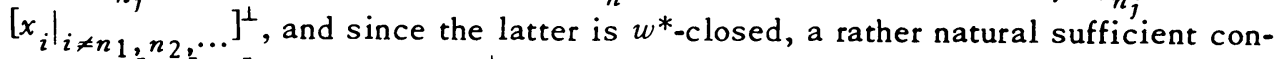
dition is $\left[f_{n_{j}}\right]=\left[\left.x_{i}\right|_{i \neq n_{1}}, n_{2}, \ldots\right]^{\perp}$. This condition is violated in Example 1 since $\sum_{i=1}^{\infty} 2^{-i} b_{2 i}$ is in $\left[x_{2 n-1}\right]^{\perp}$ but is not in $\left[f_{2 n}\right]$. These considerations lead to the following problem.

Problem 1. If $E^{*}$ is separable, is there an M-basis $\left(x_{n} ; f_{n}\right)$ for which $\left[f_{n}\right]=E^{*}$ and such that, for every subsequence of the integers, $\left[f_{n_{j}}\right]$ is $\sigma\left(E^{*}, E\right)$ closed?

Problem 2. Does every separable Banach space have an M-basis for which

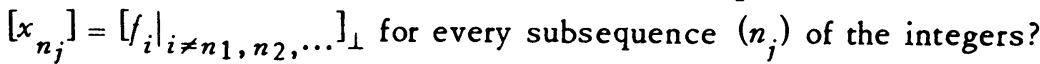

An $M$-basis satisfies the condition of Problem 2 if and only if for every $x$ in $E$ there exist multipliers $\left(\lambda_{n, i}(x) \mid 1 \leq i \leq n<\infty\right)$ such that $x=$ $\lim _{n \rightarrow \infty} \sum_{i=1}^{n} \lambda_{n, i}(x) f_{i}(x) x_{i}$. Such a condition is somewhat weaker than strongly series summable $M$-bases $([23],[14])$ in which the multipliers do not depend on $x$. Such M-bases exist in separable complex Banach spaces whose duals have the bounded approximation property [14]. 
2. On somewhat reflexive spaces. Milman [21] has also used the erroneous claim (see $\$ 1$ ) that subsequences of boundedly complete M-bases are boundedly complete in his proof of the following interesting result: If $E^{* *}$ is separable then botb $E$ and $E^{*}$ contain infinite dimensional reflexive subspaces.

W. B. Johnson and H. P. Rosenthal [16] have proven that the above statement is true. Below we still include a proof of this statement in the case that $E^{* *}$ has the approximation property, which we obtained independently. These results are used in the example concerning somewhat reflexive spaces in the weaker form we present.

Herman and Whitley [10] have called a space $E$ somewhat reflexive if every infinite dimensional subspace of $E$ contains an infinite dimensional reflexive subspace. They show, for example, that every quasi-reflexive space (i.e., $\left.\operatorname{dim} E^{* *} / E<\infty\right)$ is somewhat reflexive and note that the space $(J \times J \times \cdots)_{l_{2}}$ (with $J$ the quasi-reflexive space of James [11]) is somewhat reflexive. It should be noted that a proof of the Milman statement (above) will force $E$ (having $E^{* *}$ separable) to be somewhat reflexive: If $X$ is an infinite dimensional subspace of $E$, then $X^{* *} \sim X^{\perp \perp}$ makes $X^{* *}$ separable (where $\sim$ stands for "isomorphic to"), so that $X$ would contain an infinite dimensional reflexive subspace.

In what follows, we make frequent use of the following theorem of James [12] and Lindenstrauss [19]: For any separable B-space, E, there is a B-space $Y$ with a shrinking basis such that $E$ is a continuous image of $Y^{*}$ and such that $Y^{* *} \sim$ $Y \times E^{*}$.

Theorem 1. If $E^{* *}$ is separable and bas the approximation property, then both $E$ and $E^{*}$ are somewhat reflexive.

Proof. Let $Y$ be the space of James and Lindenstrauss above which has $Y^{* *} \sim Y \times E^{*}$. Then $Y^{* *}$ has the approximation property, and hence a boundedly complete basis by Theorem 1.4 of [15]. By Corollary 5 of [4], every infinite dimensional subspace $G$ of $E\left(\subset E^{* *} \subset Y^{* *}\right)$ contains a boundedly complete basic sequence, say $\left(x_{n}\right)$. By Proposition 2 of [4], some block basic sequence $\left(y_{n}\right)$ with respect to $\left(x_{n}\right)$ is shrinking. It follows that $\left[y_{n}\right] \subset G$ is reflexive as desired. The same argument can now be applied to $E^{*}$ since, by [8], $E^{*}$ has the approximation property.

We now turn to some questions raised in [10] concerning somewhat reflexive spaces. There the authors remarked that they did not know whether or not duals and/or quotients of somewhat reflexive spaces are somewhat reflexive. This example answers both questions in the negative:

Example 2. Let $Y$ be the space of James and Lindenstrauss above such that $c_{0}$ is a quotient of $Y^{*}$ and $Y^{* *} \sim Y \times l_{1} . Y$ and $l_{1}$ both have bases, so by 
Theorem $1, Y^{*}$ is somewhat reflexive. However, neither $l_{1}$ nor $c_{0}$ contains an infinite dimensional reflexive subspace so that this space is somewhat reflexive and has both a quotient and dual which are not somewhat reflexive. A somewhat more remarkable feature of this space $\left(Y^{*}\right)$ is that although it is separable, contains no copy of $c_{0}$ or $l_{1}$, its second dual $Y^{* * *} \sim Y^{*} \times m$ is nonseparable.

Consideration of this example has led the authors to the following question.

Problem 3. If $E$ is a separable somewhat reflexive space, is $E^{*}$ separable?

3. Boundedly complete $M$-bases. Many definitions of boundedly complete $M$-bases appear in the literature. All of these (known to the authors) reduce in the basis situation to the concept studied by Dunford and Morse [6], Alaoglu [1], Karlin [18] and James [11] (the name "boundedly complete" seems to be due to Day [3]).

The original definition asserts that a basis is boundedly complete if the boundedness of the partial sums $\sum_{i=1}^{n} a_{i} x_{i}$ forces the convergence of the series $\sum_{i=1}^{\infty} a_{i} x_{i}$. The main motivation for such a definition (at least at this point in time) is the fact that boundedly complete bases span dual spaces. The second author (in [25]) has shown that such "boundedness implies convergence" conditions are much too strong for use with $M$-bases. Therefore, for $M$-bases, the definitions in the literature are constructed so that boundedly complete $M$-bases span separable duals ([13], [21]). In this section we present a geometric definition of boundedly complete M-basis which is equivalent to the known "soft" definitions in the literature.

Definition. Let $\left(x_{n} ; f_{n}\right)$ be an M-basis for $E$. We shall call it norm-boundedly-complete if

$$
\sup _{n} \inf _{n} u=0
$$

implies the existence of $x$ in $E$ with $f_{n}(x)=a_{n}$ for all $n$. (Here $S_{n} u=$ $\left.\sum_{i=1}^{n} f_{i}(u) x_{i}.\right)$

The above definition is strongly related to the norming characteristic of the subspace $\left[f_{n}\right]$ of $E^{*}$ through the following considerations: For any $x$ in $E$ let

$$
|x|=\sup _{f \in\left[f_{n}\right] ;\|f\| \leq 1} f(x) .
$$

This always defines a norm on $E$, and in case it is equivalent to the original norm, we say that $\left[f_{n}\right]$ is norming (or of positive characteristic [5]). If $\left(x_{n}\right)$ is a basis for $E$, it follows that $\left[f_{n}\right]$ is norming [24]. If $\left(x_{n} ; f_{n}\right)$ is an M-basis for $E$, then it can be shown that 


$$
|x|=\sup _{n} \inf _{S_{n} u=0}\left\|\left(S_{n} x\right)+u\right\|
$$

In what follows, $\phi: E \rightarrow\left[f_{n}\right] *$ is to denote the natural map defined by $(\phi(x)) f$ $=f(x)$. It is well known (e.g. [5]) that $\left[f_{n}\right]$ is norming if and only if $\phi$ is an isomorphism of $E$ into $\left[f_{n}\right] *$. We recall (see the introduction) that we call an M-basis $\left(x_{n} ; f_{n}\right)$ of $E$ boundedly complete if $\phi$ is an isomorphism of $E$ into [ $\left.f_{n}\right] *$ (equiv. alently, $\left.\phi(E)=\left[f_{n}\right] *\right)$.

Theorem 2. An M-basis $\left(x_{n} ; f_{n}\right)$ for $E$ is boundedly complete if and only if it is norm-boundedly complete.

Proof. Assume that $\phi(E)=\left[f_{n}\right] *$ and let $\left(a_{j}\right)$ be a sequence of scalars with

$$
\inf _{n}\left\|\sum_{i=1}^{n} a_{i} x_{i}+u\right\|
$$

bounded in $n$. Since $\left[f_{n}\right]$ is separable, some subsequence of $\phi\left(\sum_{i=1}^{n} a_{i} x_{i}+u_{n}\right)$ converges weak* to some $F$ in $\left[f_{n}\right] *$. $\left(\left(u_{n}\right)\right.$ has been chosen to keep $\left(\sum_{i=1}^{n} a_{i} x_{i}+u_{n}\right)$ a bounded sequence.) Then $F=\phi(x)$ for some $x \in E$ whence

$$
f(x)=(\phi(x))(f)=F(f)=\lim _{k}\left(\phi\left(\sum_{i=1}^{n_{k}} a_{i} x_{i}+u_{n_{k}}\right)\right) f=\lim _{k} f\left(\sum_{i=1}^{n_{k}} a_{i} x_{i}+u_{n_{k}}\right)
$$

for all $f \in\left[f_{n}\right]$ and hence $f_{i}(x)=a_{i}(i=1,2, \ldots)$. For the other direction suppose that $\left(y_{j}\right)$ is a bounded sequence in $E$ with $\lim _{j} f_{n}\left(y_{j}\right)=a_{n}$ (existing for each $n$ ). It follows that $S_{n}\left(y_{j}\right)$ converges strongly to $\sum_{i=1}^{n} a_{i} x_{i}$ for each $n$. Thus, one can choose $\left(y_{j_{n}}\right)$ and

$$
z_{n}=\sum_{i=1}^{n} a_{i} x_{i}+\left(I-S_{n}\right) y_{j_{n}}
$$

in such a way that $\left\|z_{n}-y_{j_{n}}\right\|<2^{-n}$ for each $n$. Since

$$
\inf _{n}\left\|=0 \sum_{i=1}^{n} a_{i} x_{i}+u\right\| \leq\left\|z_{n}\right\| \leq\left\|y_{j_{n}}\right\|+2^{-n}
$$

there is $x$ in $E$ with $f_{i}(x)=a_{i}$ for all $i$. This gives the result by Theorem II.5 of [13].

4. M-bases and complemented subspaces. Milman's Proposition 3.5, Theorem 3.7, and Theorem 3.8 of [21] give conditions which guarantee the complementation in $E$ of $\left[x_{n}\right]$ where $\left(x_{n}\right)$ is a boundedly complete basic sequence in the 
separable Banach space $E$. For example, $\left[x_{n}\right]$ is complemented if there are coefficient functionals $\left(f_{n}\right) \subset E^{*}$ with $\left[f_{n}\right]$ norming $\left[x_{n}\right]$. In Theorem 3 below, $\left(x_{n_{k}}\right)$ need not be basic or boundedly complete, but must be part of an M-basis for $E$. Extensions of $M$-bases of subspaces to M-bases of $E$ are treated in [9] and [26].

Theorem 3. Let $\left(x_{n} ; f_{n}\right)$ be an M-basis for E, let $\left(n_{k}\right)$ be an infinite sub. sequence of the integers and let $\left(n_{k}^{\prime}\right)$ denote the complementary subsequence. If $\left[f_{n_{k}}\right]$ is norming over $\left[x_{n_{k}}\right]$, then $E=\left[x_{n_{k}}\right] \oplus\left[x_{n_{k}{ }^{\prime}}\right]$. If $\left[f_{n}\right]$ is norming over $E$, the converse bolds.

Proof. Consider the quotient map $q: E \rightarrow E /\left[x_{n_{k}}\right]$. Then by the norming hypothesis and by $\left[f_{n_{k}}\right] \subset\left[x_{n_{k}^{\prime}}\right]^{\perp}$, there exists $\mu>0$ such that, for $u \in\left[x_{n_{k}}\right]$,

$$
\mu\|u\| \leq \sup _{g \in\left[f_{n_{k}}\right] ;\|g\| \leq 1}|g(u)| \leq\|q(u)\| \leq\|u\|,
$$

whence $q \mid\left[x_{n_{k}}\right]$ is an isomorphism. Further $\operatorname{sp}\left(x_{n}\right)$ is dense in $E$, so that $\operatorname{sp}\left(q\left(x_{n_{k}}\right)\right)$ is dense in $E /\left[x_{n_{k}}\right]$. Thus, $\left[x_{n_{k}}\right]$ is isomorphic to $E /\left[x_{n_{k}}\right]$. It is standard (and readily verified) that a projection of $E$ onto $\left[x_{n_{k}}\right]$ along $\left[x_{n_{k}{ }^{1}}\right]$ is given by $P=\left(q \mid\left[x_{n_{k}}\right]\right)^{-1} q$.

For the second assertion, let $\left[f_{n}\right]$ be norming over $E=\left[x_{n_{k}}\right] \oplus\left[x_{n_{k}}\right]$. Let $u \in\left[x_{n_{k}}\right]$ and $g \in \operatorname{sp}\left(f_{n}\right)$ with $\|g\| \leq 1$ such that $g(u) \geq \mu\|u\|$. If $P$ is the projection of $E$ onto $\left[x_{n_{k}}\right]$ along $\left[x_{n_{k}}\right], P^{*} g \in \operatorname{sp}\left(f_{n_{k}}\right)$ (because $P^{*} f_{n_{k}}=f_{n_{k}}$, $\left.P^{*} f_{n_{k}}=0\right)$ and $\left\|P^{*}\right\|\left(P^{*} g /\left\|P^{*}\right\|\right)(u)=\left(P^{*} g\right)(u)=g(P u)=g(u) \geq \mu\|u\|$. Thus, $\left(P^{*} g /\|P\|\right)(u) \geq(\mu /\|P\|)\|u\|$, so that $\left[f_{n_{k}}\right]$ norms $\left[x_{n_{k}}\right]$.

Corollary. Let $\left(x_{n} ; f_{n}\right)$ be an M-basis for $E$ sucb that for every subsequence $\left(n_{k}\right)$ of the integers, $\left[f_{n_{k}}\right]$ norms $\left[x_{n_{k}}\right]$. Then $\left(x_{n}\right)$ is an unconditional bas is of $E$.

Proof. For every $\left(n_{k}\right)$, by Theorem $3, E=\left[x_{n_{k}}\right] \oplus\left[x_{n_{k}}\right]$. The result follows from a result of Lorch [20].

\section{REFERENCES}

1. L. Alaoglu, Weak topologies of normed linear spaces, Ann. of Math. (2) 41 (1940), 252-267. MR 1, 241.

2. S. Banach, Théorie des opérations linéaires, Monografie Mat., PWN, Warsaw, 1932.

3. M. M. Day, Normed linear spaces, Ergebnisse der Mathematik und ihrer Grenzgebiete, N. F., Heft 21, Reihe: Reelle Funktionen, Springer-Verlag, Berlin, 1958.

MR $20 \# 1187$.

4. D. W. Dean, I. Singer and L. Sternbach, On shrinking basic sequences in Banach spaces, Studia Math, 40 (1971), 23-33.

5. J. Dixmier, Sur un théorème de Banach, Duke Math J. 15 (1948), 1057-1071. MR 10, 306 . 
6. N. Dunford and A. P. Morse, Remarks on the preceding paper of James A. Clarkson, Trans. Amer. Math. Soc. 40 (1936), 415-420.

7. V. F. Gapoškin and M. I. Kadec, Operator bases in Banach space, Mat. Sb. 61 (103) (1963), 2-12. (Russian) MR $27 \# 1810$.

8. A. Grothendieck, Produits tensoriels topologiques et espaces nucléaires, Mem. Amer. Math. Soc. No. 16 (1955). MR 17, 763.

9. V. I. Gurarǐ and M. I. Kadec, Minimal systems and quasicomplements in Banach space, Dokl. Akad. Nauk SSSR 145 (1962), 255-258 = Soviet Math. Dokl. 3 (1962), 966968. MR $26 \# 6728$.

10. R. Herman and R. Whitley, An example conceming reflexivity, Studia Math. 28 (1966/67), 289-294. MR $35 \# 5900$.

11. R. C. James, Bases and reflexivity of Banach spaces, Ann. of Math. (2) 52 (1950), 518-527. MR 12, 616.

12. - Separable conjugate spaces, Pacific J. Math. 10 (1960), 563-571. MR $22 \# 8307$.

13. W. B. Johnson, Markushevich bases and duality theory, Trans. Amer. Math. Soc. 149 (1970), 171-177. MR $41 \# 5927$.

14. - On the existence of strongly series summable Markushevich bases in Banach spaces, Trans. Amer. Math. Soc. 157 (1971), 481-486.

15. W. B. Johnson, H. P. Rosenthal and M. Zippin, On bases, finite dimensional decompositions and weaker structures in Banach spaces, Israel J. Math. 9 (1971), 488-506. MR $43 \# 6702$.

16. W. B. Johnson and H. P. Rosenthal, $O_{n} w^{*}$-basic sequences and their applications to the study of Banach spaces, Studia Math. (to appear).

17. M. I. Kadec and A. PeXczyński, Basic sequences, bi-orthogonal systems and norming sets in Banach and Fréchet spaces, Studia Math. 25 (1965), 297-323. (Russian) MR $31 \# 6112$.

18. S. Karlin, Bases in Banach spaces, Duke Math. J. 15 (1948), 971-985. MR 10,548.

19. J. Lindenstrauss, On James' paper "Separable conjugate spaces", Israel J. Math. 9 (1971), 279-284. MR 43 \#5289.

20. E. R. Lorch, Bicontinuous linear transformations in certain vector spaces, Bull. Amer. Math. Soc. 45 (1939), 564-569. MR 1, 58.

21. V. D. Mil'man, The geometric theory of Banach spaces, Uspehi Mat. Nauk 25 (1970), no. 3 (153), 132-138. (Russian)

22. A. Pelczyński, Some problems on bases in Banach and Fréchet spaces, Israel J. Math. 2 (1964), 132-138. MR $30 \# 3356$.

23. W. Ruckle, Representation and series summability of complete biorthogonal sequences, Pacific J. Math. 34 (1970), 511-528. MR $42 \sharp 2219$.

24. I. Singer, On the constants of basic sequences in Banach spaces, Studia Math. 31 (1968), 125-134. MR $38 \# 6338$.

25. - - Bases in Ban ach spaces. II, Stud. i Cerc. Mat. 15 (1964), 157-208. (Romanian) MR $32 \sharp 8121$ b.

26. - On biorthogonal systems and total sequences of functionals, Math. Ann. 193 (1971), 183-188.

DEPARTMENT OF MATHEMATICS, OHIO STATE UNIVERSITY, COLUMBUS, OHIO 43210

INSTITUTE OF MATHEMATICS, ACADEMY OF SCIENCE OF THE SOCIALIST REPUBLIC OF ROMANIA, BUCHAREST, ROMANIA 